\title{
Fast and accurate numerical methods for solving elliptic difference equations defined on lattices
}

\author{
A. Gillman, P.G. Martinsson \\ Department of Applied Mathematics, 526 UCB, University of Colorado, Boulder, CO 80309
}

\begin{abstract}
Techniques for rapidly computing approximate solutions to elliptic PDEs such as Laplace's equation are well established. For problems involving general domains, and operators with constant coefficients, a highly efficient approach is to rewrite the boundary value problem as a boundary integral equation (BIE), and then solve the BIE using fast methods such as, e.g., the Fast Multipole Method (FMM). The current paper demonstrates that this procedure can be extended to elliptic difference equations defined on infinite lattices, or on finite lattice with boundary conditions of either Dirichlet or Neumann type. As a representative model problem, a lattice equivalent of Laplace's equation on a square lattice in two dimensions is considered: discrete analogs of BIEs are derived and fast solvers analogous to the FMM are constructed. Fast techniques are also constructed for problems involving lattices with inclusions and local deviations from perfect periodicity. The complexity of the methods described is $O\left(N_{\text {boundary }}+N_{\text {source }}+N_{\text {inc }}\right)$ where $N_{\text {boundary }}$ is the number of nodes on the boundary of the domain, $N_{\text {source }}$ is the number of nodes subjected to body loads, and $N_{\text {inc }}$ is the number of nodes that deviate from perfect periodicity. This estimate should be compared to the $O\left(N_{\text {domain }} \log N_{\text {domain }}\right)$ estimate for FFT based methods, where $N_{\text {domain }}$ is the total number of nodes in the lattice (so that in two dimensions, $N_{\text {boundary }} \sim N_{\text {domain }}^{1 / 2}$ ). Several numerical examples are presented.
\end{abstract}

Keywords: difference equation, fast convolution, discrete Laplace operator, boundary integral equation, fast solver, fast direct solver, fast multipole method, hierarchically semi-separable matrix

\section{Introduction}

The paper describes efficient techniques for solving elliptic difference equations defined either on the integer lattices $\mathbb{Z}^{2}$ or $\mathbb{Z}^{3}$, or on finite sub-domains of those lattices. The techniques are applicable to a wide range of difference equations, but to keep the presentation simple, we focus on problems in two dimensions involving

Email address: adrianna.gillman@colorado.edu, martinss@colorado.edu (A. Gillman, P.G. Martinsson ) 
the well known discrete Laplace operator A which for $\boldsymbol{m} \in \mathbb{Z}^{2}$ takes the form

$$
[\mathrm{A} u](\boldsymbol{m})=4 u(\boldsymbol{m})-u\left(\boldsymbol{m}+\boldsymbol{e}_{1}\right)-u\left(\boldsymbol{m}-\boldsymbol{e}_{1}\right)-u\left(\boldsymbol{m}+\boldsymbol{e}_{2}\right)-u\left(\boldsymbol{m}-\boldsymbol{e}_{2}\right) .
$$

In (1.1), $\boldsymbol{e}_{1}=[1,0]$ and $\boldsymbol{e}_{2}=[0,1]$ are the canonical basis vectors in $\mathbb{Z}^{2}$, and $u=u(\boldsymbol{m})$ is a real valued function on $\mathbb{Z}^{2}$. We will describe techniques for both the free space equation

$$
[\mathrm{A} u](\boldsymbol{m})=f(\boldsymbol{m}), \quad \boldsymbol{m} \in \mathbb{Z}^{2}
$$

and for boundary value problems of the form

$$
\left\{\begin{aligned}
{[\mathrm{A} u](\boldsymbol{m}) } & =0, & & \boldsymbol{m} \in \Omega, \\
u(\boldsymbol{m}) & =g(\boldsymbol{m}), & & \boldsymbol{m} \in \Gamma .
\end{aligned}\right.
$$

In (1.3), $\Omega$ is a subset of $\mathbb{Z}^{2}$ with boundary $\Gamma$. A precise definition of what we mean by the boundary of a lattice domain is given in Section 4 . We typically refer to the data function $f$ as a source, and the unknown function $u$ as a potential.

Equations of the forms (1.2) and (1.3) are perhaps best known as equations arising upon finite difference discretizations of Poisson's and Laplace's equations, but they arise naturally in a wide range of applications. To name just a few examples, equations (1.2) or (1.3) or variations of them appear directly in models of random walks [1], in analyzing the Ising model, in determining vibration modes of crystals, and in modelling QCD $[2,3]$. Additional examples arise in engineering mechanics as micro-structural models, macroscopic models, simulating fractures [4, 5] and as models of periodic truss and frame structures $[6,7,8,9]$.

The techniques described in this paper can be viewed as an adaptation to the discrete case of a set of analytical and numerical methods for efficiently solving the corresponding continuum equations. For instance, equation (1.2) has a continuum analog in the free space Laplace equation

$$
[-\Delta u](\boldsymbol{x})=f(\boldsymbol{x}), \quad \boldsymbol{x} \in \mathbb{R}^{2},
$$

coupled with suitable decay conditions at infinity. The analytic solution of (1.4) is

$$
u(\boldsymbol{x})=[\Phi * f](\boldsymbol{x})=\int_{\mathbb{R}^{2}} \Phi(\boldsymbol{x}-\boldsymbol{y}) f(\boldsymbol{y}) d A(\boldsymbol{y}),
$$

were $\Phi$ is the fundamental solution of the Laplace operator,

$$
\Phi(\boldsymbol{x})=-\frac{1}{2 \pi} \log |\boldsymbol{x}|
$$

When $f$ is compactly supported, the integral in (1.5) can be discretized using appropriate quadratures, and the resulting finite sum can be evaluated rapidly via, e.g., the Fast Multipole Method (FMM). In the discrete case, it turns out to be possible to define a lattice fundamental function $\phi$ such that the exact solution of (1.2) is

$$
u(\boldsymbol{m})=[\phi * f](\boldsymbol{m})=\sum_{\boldsymbol{n} \in \mathbb{Z}^{2}} \phi(\boldsymbol{m}-\boldsymbol{n}) f(\boldsymbol{n}) .
$$


The function $\phi$ cannot be expressed directly in terms of elementary functions, but can easily be evaluated numerically, for instance from its Fourier representation

$$
\phi(\boldsymbol{m})=\frac{1}{(2 \pi)^{2}} \int_{[-\pi, \pi]^{2}} \frac{\cos (\boldsymbol{m} \cdot \boldsymbol{t})-1}{4 \sin ^{2}\left(t_{1} / 2\right)+4 \sin ^{2}\left(t_{2} / 2\right)} d A(\boldsymbol{t}), \quad \boldsymbol{m} \in \mathbb{Z}^{2} .
$$

In Section 2, we demonstrate that when $f$ is supported on a finite number $N_{\text {source }}$ of source points, the sum (1.6) can be evaluated rapidly via a lattice version of the FMM. Supposing that the potential is required only at the source points, the computational cost $T_{\text {freespace }}$ of the scheme satisfies

$$
T_{\text {freespace }} \sim N_{\text {source }}
$$

The techniques for the free space problem on a perfectly periodic infinite lattice can easily be modified to handle local deviations from periodicity. Specifically, we describe in Section 3 techniques for solving the equation

$$
[(\mathrm{A}+\mathrm{B}) u](\boldsymbol{m})=f(\boldsymbol{m}), \quad \boldsymbol{m} \in \mathbb{Z}^{2},
$$

where $\mathrm{B}$ is a local operator acting on some finite subset $\Omega_{\text {inc }} \subset \mathbb{Z}^{2}$. An equation such as (1.8) can be used to model a lattice in which a finite number of bars have been added or removed, or have had their conductivities changed, see Figure 3.1. By convolving the equation (1.8) by the lattice fundamental solution $\phi$, an equation defined on the finite subset $\Omega_{\text {inc }}$ is obtained. Moreover, this equation can using fast methods be solved in time $T_{\text {inc }}$, where $T_{\text {inc }}$ scales linearly with the number of points $N_{\text {inc }}$ in the inclusion,

$$
T_{\text {inc }} \sim N_{\text {inc }}+N_{\text {source }}
$$

For boundary value problems such as (1.3), the techniques proposed in this manuscript are lattice analogs of Boundary Integral Equation (BIE) methods for solving elliptic partial differential equations. To illustrate, let us consider a Laplace boundary value problem with Dirichlet boundary conditions that is a continuum analog of (1.3):

$$
\left\{\begin{aligned}
{[-\Delta u](\boldsymbol{x}) } & =0, & & \boldsymbol{x} \in \Omega, \\
u(\boldsymbol{x}) & =g(\boldsymbol{x}), & & \boldsymbol{x} \in \Gamma .
\end{aligned}\right.
$$

It is possible to reduce (1.10) to an equation on $\Gamma$ by first representing the solution $u$ as a double layer potential

$$
u(\boldsymbol{x})=\int_{\Gamma} D(\boldsymbol{x}, \boldsymbol{y}) \sigma(\boldsymbol{y}) d \ell(\boldsymbol{y}),
$$

where $D$ is the so called double layer kernel,

$$
D(\boldsymbol{x}, \boldsymbol{y})=\frac{\partial}{\partial \boldsymbol{n}(\boldsymbol{y})} \Phi(\boldsymbol{x}-\boldsymbol{y})=\boldsymbol{n}(\boldsymbol{y}) \cdot \nabla_{\boldsymbol{y}} \Phi(\boldsymbol{x}-\boldsymbol{y})=\frac{\boldsymbol{n}(\boldsymbol{y}) \cdot(\boldsymbol{x}-\boldsymbol{y})}{2 \pi|\boldsymbol{x}-\boldsymbol{y}|^{2}}
$$

where $\boldsymbol{n}(\boldsymbol{y})$ is the unit normal vector of $\Gamma$ at $\boldsymbol{y}$. The boundary density function $\sigma$ in (1.10) is determined by calculating the limit of $u(\boldsymbol{x})$ as $\boldsymbol{x}$ approaches the boundary 
$\Gamma$ and equating the result with the Dirichlet condition in (1.10). This results in the second kind Fredholm equation

$$
\frac{1}{2} \sigma(\boldsymbol{x})+\int_{\Gamma} D(\boldsymbol{x}, \boldsymbol{y}) \sigma(\boldsymbol{y}) d \ell(\boldsymbol{y})=g(\boldsymbol{x}), \quad \boldsymbol{x} \in \Gamma .
$$

We find that a solution to (1.10) can now be obtained by solving the equation (1.12). The reformulation offers several advantages, including a reduction in dimensionality, and a well conditioned equation [10]. An apparent disadvantage of using (1.12) instead of (1.10) as a foundation for numerical work is that (1.12) leads to dense systems upon discretization. This potential drawback can again be overcome by the FMM. A principal contribution of the present paper is to demonstrate that existing fast numerical methods developed for continuum problems can be modified to work for lattice equations and lead to highly efficient solvers. It has been demonstrated $[11,8]$ that the solution of $(1.3)$ can be written

$$
u(\boldsymbol{m})=\sum_{n \in \Gamma} d(\boldsymbol{m}, \boldsymbol{n}) \sigma(\boldsymbol{n}),
$$

where $d$ is a discrete analog of the continuum double layer potential (1.12). (Equation (4.11) provides the precise definition.) An equation for $\sigma$ is obtained by inserting (1.13) into the boundary condition in (1.3) which results in the boundary equation

$$
\sum_{n \in \Gamma} d(\boldsymbol{m}, \boldsymbol{n}) \sigma(\boldsymbol{n})=g(\boldsymbol{m}), \quad \boldsymbol{m} \in \Gamma
$$

It was shown in [11] that (1.14) is very well conditioned (as the continuum analog would indicate) and we demonstrate in Section 4 that it can be solved in time

$$
T_{\text {bvp }} \sim N_{\text {boundary }},
$$

where $N_{\text {boundary }}$ denotes the number of points in $\Gamma$.

The techniques for handling (i) body loads, (ii) deviations from periodicity, and (iii) boundary conditions, can all be combined. We demonstrate in Section 5 that an equation of the form

$$
\left\{\begin{aligned}
{[(\mathrm{A}+\mathrm{B}) u](\boldsymbol{m}) } & =f(\boldsymbol{m}), & & \boldsymbol{m} \in \Omega, \\
u(\boldsymbol{m}) & =g(\boldsymbol{m}), & & \boldsymbol{m} \in \Gamma,
\end{aligned}\right.
$$

can be solved in time $T_{\text {combined }}$ that satisfies

$$
T_{\text {combined }} \sim N_{\text {source }}+N_{\text {inc }}+N_{\text {boundary }} .
$$

The core point of the paper is that the time requirements (1.7), (1.9), (1.15), and (1.17) are in a strong sense optimal: The computational time depends linearly on the amount of actual input data. In contrast, the conventional approach to solve, e.g., the boundary value problem (1.3) would be to form and solve a linear system of size $N_{\text {domain }} \times N_{\text {domain }}$, where $N_{\text {domain }}$ denotes the number of points in $\Omega$. This system 
is sparse, and can often be solved in time proportional to the number of degrees of freedom (using, e.g., multigrid). However, the time $T_{\text {conventional }}$ required even for such a linear complexity solver would satisfy

$$
T_{\text {conventional }} \sim N_{\text {domain }},
$$

which is far worse that (1.15) since one would typically have

$$
\begin{array}{ll}
N_{\text {domain }} \sim N_{\text {boundary }}^{2} & \text { when } \Omega \subset \mathbb{Z}^{2}, \\
N_{\text {domain }} \sim N_{\text {boundary }}^{3 / 2} & \text { when } \Omega \subset \mathbb{Z}^{3} .
\end{array}
$$

In fairness, it must be noted that the constant of proportionality in the estimate (1.18) for conventional methods is typically lower than that in (1.15) for the methods proposed here. In particular, there exist very fast methods based on the FFT which exploit the fact that a constant coefficient difference equation on a regular grid is diagonal in Fourier space. The time $T_{\mathrm{fft}}$ required by such a method satisfies

$$
T_{\mathrm{fft}} \sim N_{\text {domain }} \log N_{\text {domain }},
$$

but with a very small constant. A limitation of FFT based methods is that they intrinsically require the computational domain to be a rectangle with periodic boundary conditions. However, they are so fast that even for problems involving other boundary conditions, it often makes sense to either modify the mathematical model to conform to the available numerical tool, or to implement various "correction" techniques. In contrast, the methods proposed in this paper have the advantage of being able to naturally handle domains of different shapes and with different boundary conditions (Dirichlet, Neumann, decay at infinity, periodic or quasi-periodic, etc), in addition to their advantage of having complexity $O\left(N_{\text {boundary }}\right)$ rather than $O\left(N_{\text {domain }}\right)$.

Some indication of the problem size at which it becomes advantageous to switch to the methods proposed in this paper is given by the numerical examples reported in Section 6. The switching point depends on the computational environment, but typically occurs for lattices with between $10^{4}$ and $10^{6}$ nodes.

\section{Techniques for the free space problem}

This section describes a fast numerical method for solving a lattice analog of a free space Poisson equation.

\subsection{Problem definition}

With A defined by (1.1), the free space lattice Poisson equation for a given source function $f$ takes the form

$$
[\mathrm{A} u](\boldsymbol{m})=f(\boldsymbol{m}), \quad \boldsymbol{m} \in \mathbb{Z}^{2} .
$$

We assume for simplicity that $f$ has compact support. In this case, the equation (2.1) has a unique solution that tends to zero at infinity whenever

$$
\sum_{\boldsymbol{m} \in \mathbb{Z}^{2}} f(\boldsymbol{m})=0
$$


If (2.2) does not hold, then we require $u$ to grow at most logarithmically at infinity,

$$
\sup _{\boldsymbol{m} \in \mathbb{Z}^{2}} \frac{|u(\boldsymbol{m})|}{\log (2+|\boldsymbol{m}|)}<\infty,
$$

which ensures that (2.1) has a unique solution (up to a shift by a constant), see [8].

\subsection{Continuum analog}

A continuum analog of (2.1) is the Poisson equation

$$
[-\Delta u](\boldsymbol{x})=f(\boldsymbol{x}), \quad \boldsymbol{x} \in \mathbb{R}^{2},
$$

whose analytic solution (under suitable decay conditions on $f$ and $u$ ) is

$$
u(\boldsymbol{x})=[\Phi * f](\boldsymbol{x})=\int_{\mathbb{R}^{2}} \Phi(\boldsymbol{x}-\boldsymbol{y}) f(\boldsymbol{y}) d A(\boldsymbol{y}),
$$

where $\Phi$ is the fundamental solution of the Laplace operator,

$$
\Phi(\boldsymbol{x})=-\frac{1}{2 \pi} \log |\boldsymbol{x}|
$$

\subsection{The lattice fundamental solution}

As mentioned in the introduction, it is possible to construct a fundamental solution $\phi$ to the discrete Laplacian such that a solution to (2.1) and (2.3) is provided by the convolution

$$
u(\boldsymbol{m})=[\phi * f](\boldsymbol{m})=\sum_{\boldsymbol{n} \in \mathbb{Z}^{2}} \phi(\boldsymbol{m}-\boldsymbol{n}) f(\boldsymbol{n}) .
$$

In this section, we define $\phi$, sketch how to construct it, and describe its asymptotic expansion at infinity.

\subsubsection{Definition}

The lattice fundamental solution is defined as the unique function $\phi$ that satisfies the three conditions

$$
\begin{aligned}
\sup _{\boldsymbol{m} \in \mathbb{Z}^{2}} \frac{|\phi(\boldsymbol{m})|}{\log (2+|\boldsymbol{m}|)} & <\infty \\
\phi(\mathbf{0}) & =0, \\
\mathrm{~A} \phi & =\delta
\end{aligned}
$$

where the function $\delta$ is defined by

$$
\delta(\boldsymbol{m})= \begin{cases}1, & \boldsymbol{m}=0 \\ 0, & \boldsymbol{m} \neq 0 .\end{cases}
$$

For future reference, we define the solution operator of (2.1) and (2.3) as the operator $\mathrm{G}$ defined via

$$
[G f](\boldsymbol{m})=[\phi * f](\boldsymbol{m})=\sum_{n \in \mathbb{Z}^{2}} \phi(\boldsymbol{m}-\boldsymbol{n}) f(\boldsymbol{n}) .
$$

Then the solution to (2.1) and (2.3) is simply $u=\mathrm{G} f$. 


\subsubsection{Analytic formula}

In order to construct an analytic formula for $\phi$, we introduce the discrete Fourier transform $F$ via

$$
\hat{u}(\boldsymbol{t})=[F u](\boldsymbol{t})=\sum_{\boldsymbol{m} \in \mathbb{Z}^{2}} e^{i \boldsymbol{m} \cdot \boldsymbol{t}} u(\boldsymbol{m}), \quad \boldsymbol{t} \in[-\pi, \pi]^{2} .
$$

The inverse transform is given by

$$
u(\boldsymbol{m})=\left[F^{*} \hat{u}\right](\boldsymbol{m})=\frac{1}{(2 \pi)^{2}} \int_{[-\pi, \pi]^{2}} e^{-i \boldsymbol{m} \cdot \boldsymbol{t}} \hat{u}(\boldsymbol{t}) d A(\boldsymbol{t}), \quad \boldsymbol{m} \in \mathbb{Z}^{2} .
$$

With (2.10) and (2.11), the discrete Laplace operator has the Fourier representation

$$
\left[F \mathbf{A} F^{*} \hat{u}\right](\boldsymbol{t})=4 \hat{u}(\boldsymbol{t})-e^{i t_{1}} \hat{u}(\boldsymbol{t})-e^{-i t_{1}} \hat{u}(\boldsymbol{t})-e^{i t_{2}} \hat{u}(\boldsymbol{t})-e^{-i t_{2}} \hat{u}(\boldsymbol{t})=\sigma(\boldsymbol{t}) \hat{u}(\boldsymbol{t}),
$$

where the symbol $\sigma$ of $\mathrm{A}$ is given by

$$
\sigma(\boldsymbol{t})=4-e^{i t_{1}}-e^{-i t_{1}}-e^{i t_{2}}-e^{-i t_{2}}=4 \sin ^{2} \frac{t_{1}}{2}+4 \sin ^{2} \frac{t_{2}}{2} .
$$

Applying $F$ to both sides of (2.7), we get the equation

$$
\sigma(\boldsymbol{t}) \hat{\phi}(\boldsymbol{t})=1 \text {. }
$$

It seems that $\phi$ should now be obtained by simply solving for $\hat{\phi}$ and applying the inverse Fourier transform. This would lead to the formula

$$
\phi(\boldsymbol{m})=\frac{1}{(2 \pi)^{2}} \int_{[-\pi, \pi]^{2}} e^{-i \boldsymbol{m} \cdot \boldsymbol{t}} \frac{1}{\sigma(\boldsymbol{t})} d A(\boldsymbol{t}) .
$$

However, the integrand in (2.12) is strongly singular, and must be renormalized. The result is the formula

$$
\phi(\boldsymbol{m})=\frac{1}{(2 \pi)^{2}} \int_{[-\pi, \pi]^{2}} \frac{e^{-i \boldsymbol{m} \cdot \boldsymbol{t}}-1}{\sigma(\boldsymbol{t})} d A(\boldsymbol{t}) .
$$

See $[8,12]$ for details.

\subsubsection{Asymptotic expansion}

It has been established (see e.g. $[8,12,13,14,15]$ ) that as $|\boldsymbol{m}| \rightarrow \infty$, the fundamental solution $\phi$ defined by (2.13) has the asymptotic expansion

$$
\begin{aligned}
& \phi(\boldsymbol{m})=-\frac{1}{2 \pi}\left(\log |\boldsymbol{m}|+\gamma+\frac{\log 8}{2}\right)+\frac{1}{24 \pi} \frac{m_{1}^{4}-6 m_{1}^{2} m_{2}^{2}+m_{2}^{4}}{|\boldsymbol{m}|^{6}} \\
& +\frac{1}{480 \pi} \frac{43 m_{1}^{8}-772 m_{1}^{6} m_{2}^{2}+1570 m_{1}^{4} m_{2}^{4}-772 m_{1}^{2} m_{2}^{6}+43 m_{2}^{8}}{|\boldsymbol{m}|^{12}}+O\left(1 /|\boldsymbol{m}|^{6}\right) .
\end{aligned}
$$

The number $\gamma$ is the Euler constant $(\gamma=0.577206 \cdots)$. 


\subsection{Fast evaluation of convolutions via the Fast Multipole Method}

In this section we describe certain modifications to the classical Fast Multipole Method (FMM) [16, 17] that allow the rapid evaluation of the lattice potential due to a set of sources $\left\{f_{i}\right\}_{i=1}^{N_{\text {source }}}$, placed at points $\left\{\boldsymbol{n}_{i}\right\}_{i=1}^{N_{\text {source }}} \subset \mathbb{Z}^{2}$. Assuming that we seek the potential at the locations of the sources, we need to evaluated the sums

$$
u_{i}=\sum_{j=1}^{N_{\text {source }}} f_{j} \phi\left(\boldsymbol{n}_{i}-\boldsymbol{n}_{j}\right), \quad i=1,2, \ldots, N_{\text {source }}
$$

Our first step is to split the sum into a near-field and a far-field term. We say that two sources $i$ and $j$ are near if $\left|\boldsymbol{n}_{i}-\boldsymbol{n}_{j}\right|_{\infty} \leq L$, where $|\cdot|_{\infty}$ denotes the $\ell^{\infty}$ norm on $\mathbb{Z}^{2}$, and where $L$ is an adjustable parameter. The sum (2.15) then splits into two parts

$$
u_{i}=u_{i}^{\text {near }}+u_{i}^{\text {far }},
$$

where the near-field is defined by

$$
u_{i}^{\text {near }}=\sum_{j:\left|\boldsymbol{n}_{i}-\boldsymbol{n}_{j}\right|_{\infty} \leq L} f_{j} \phi\left(\boldsymbol{n}_{i}-\boldsymbol{n}_{j}\right), \quad i=1,2, \ldots, N_{\text {source }}
$$

and the far-field is defined by

$$
u_{i}^{\mathrm{far}}=\sum_{j:\left|\boldsymbol{n}_{i}-\boldsymbol{n}_{j}\right|_{\infty}>L} f_{j} \phi\left(\boldsymbol{n}_{i}-\boldsymbol{n}_{j}\right), \quad i=1,2, \ldots, N_{\text {source }}
$$

We first observe that for each lattice node there are only $4 L^{2}+4 L$ other nodes in its near-field. The values of the lattice fundamental solution for these possible interactions can be pre-computed by directly evaluating the integral (2.13) and storing the results (due to symmetries, only $(L+1)(L+2) / 2$ values are actually needed). The near-field contribution can therefore be evaluated to floating-point precision using, at worst, $O\left(N L^{2}\right)$ operations.

For the far-field contribution, we approximate the sum (2.17) by replacing the kernel $\phi$ by an approximation $\phi_{\text {far }}$ obtained by omitting the $O\left(|\boldsymbol{m}|^{-6}\right)$ term in (2.14). The introduced error is controlled by the parameter $L$; we found that by choosing $L=30$, the relative error incurred was less than $10^{-12}$. The resulting sum

$$
u_{i}^{\mathrm{far}} \approx \sum_{j:\left|\boldsymbol{n}_{i}-\boldsymbol{n}_{j}\right|_{\infty}>L} f_{j} \phi_{\mathrm{far}}\left(\boldsymbol{n}_{i}-\boldsymbol{n}_{j}\right), \quad i=1,2, \ldots, N_{\text {source }}
$$

is then amenable to the either the classical FMM [16, 17], or more recent kernelindependent variations $[18,19,20]$. We chose to implement a two-dimensional version of the method of [19] since it was readily available. For a problem on $\mathbb{Z}^{3}$, we would expect the FFT-accelerated method of [20] to perform better. 


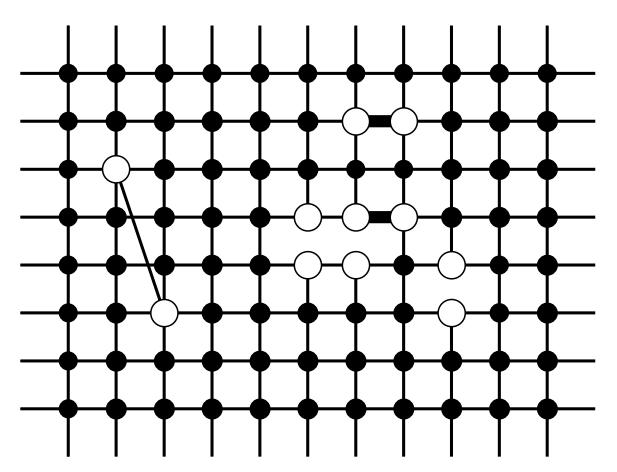

Figure 3.1: A piece of an infinite lattice with some deviations from perfect periodicity. One bar has been added, three bars have been removed, and two bars have been strengthened. The set $\Omega_{\mathrm{inc}}$ of effected nodes has 11 elements, which are marked with white circles.

\section{Techniques for lattices with inclusions}

This section describes techniques for solving a Poisson equation similar to (2.1) but with the twist that parts of the lattice may be perturbed from perfect periodicity. The mathematical model we consider can handle both the removal of links from the lattice, and the inclusion of additional ones; the only essential assumptions are that the perturbation be linear, and that only finitely many lattice nodes are affected.

\subsection{Problem definition}

We consider a perturbed lattice equation

$$
[(\mathrm{A}+\mathrm{B}) u](\boldsymbol{m})=f(\boldsymbol{m}), \quad \boldsymbol{m} \in \mathbb{Z}^{2}
$$

along with the decay condition (2.3) where B is a perturbation to the discrete Laplace operator arising from local deviations from perfect periodicity. Specifically, we assume that $B$ is such that $A+B$ remains coercive (when coupled with the decay condition $(2.3)$ ), and also that $B$ is "local" in the sense that there exists a finite set $\Omega_{\text {inc }} \subset \mathbb{Z}^{2}$ such that:

- $\mathrm{B} u=0$ when $u$ is such that $u(\boldsymbol{m})=0$ for all $\boldsymbol{m} \in \Omega_{\text {inc }}$.

- For any $u,[\mathrm{~B} u](\boldsymbol{m})=0$ when $\boldsymbol{m} \notin \Omega_{\text {inc }}$.

The two conditions amount to an assumption that B is a block diagonal operator supported on the block corresponding to $\Omega_{\text {inc }}$.

Example: Let us consider a lattice that is perturbed by adding $J$ bars to the lattice.

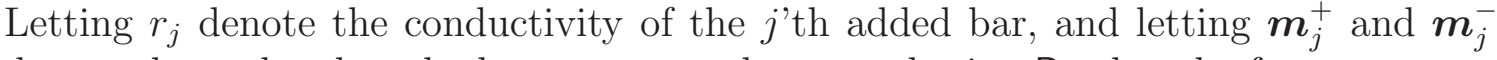
denote the nodes that the bar connects, the perturbation $B$ takes the form

$$
[\mathrm{B} u](\boldsymbol{m})=\sum_{j: \boldsymbol{m}=\boldsymbol{m}_{j}^{+}} r_{j}\left(u\left(\boldsymbol{m}_{j}^{+}\right)-u\left(\boldsymbol{m}_{j}^{-}\right)\right)+\sum_{j: \boldsymbol{m}_{=\boldsymbol{m}_{j}^{-}}} r_{j}\left(u\left(\boldsymbol{m}_{j}^{-}\right)-u\left(\boldsymbol{m}_{j}^{+}\right)\right) .
$$


In this case,

$$
\Omega_{\text {inc }}=\bigcup_{j=1}^{J}\left\{\boldsymbol{m}_{j}^{+}, \boldsymbol{m}_{j}^{-}\right\} .
$$

If all the numbers $r_{j}$ are non-negative, then the operator B defined by (3.2) is nonnegative as well, and (3.1) coupled with (2.3) has a unique solution for any $f$. The operator $\mathrm{A}+\mathrm{B}$ may be coercive even when some of the numbers $r_{j}$ are negative. For instance, if the pairs of nodes $\left\{\boldsymbol{m}_{j}^{+}, \boldsymbol{m}_{j}^{-}\right\}_{j=1}^{J}$ are connected in the original lattice, then by setting $r_{j}=-1$ for all $j$, the equations (3.1), (3.2), (2.3) model the lattice obtained by cutting the connections between the pairs $\left\{\boldsymbol{m}_{j}^{+}, \boldsymbol{m}_{j}^{-}\right\}$. Whether $\mathrm{A}+\mathrm{B}$ remains coercive now depends on the topology of the lattice after the cuts - essentially on whether all nodes remain connected.

\subsection{Continuum analog}

Equation (3.1) can be viewed as a discrete version of the perturbed free space Poisson equation

$$
-\Delta u(\boldsymbol{x})-\nabla \cdot(b(\boldsymbol{x}) \nabla u(\boldsymbol{x}))=f(\boldsymbol{x}), \quad \boldsymbol{x} \in \mathbb{R}^{2},
$$

where $b(\boldsymbol{x})$ is a function that is non-zero only in some bounded domain $\Omega_{\text {inc }}$. Now convolving (3.4) by $\Phi$, we obtain the new equation

$$
u(\boldsymbol{x})-\int_{\mathbb{R}^{2}} \Phi(\boldsymbol{x}-\boldsymbol{y}) \nabla \cdot(b(\boldsymbol{y}) \nabla u(\boldsymbol{y})) d A(\boldsymbol{y})=[\Phi * f](\boldsymbol{x}), \quad \boldsymbol{x} \in \mathbb{R}^{2} .
$$

Since $b(\boldsymbol{y})=0$ whenever $\boldsymbol{y} \notin \Omega_{\text {inc }}$, we can restrict the domain of integration in (3.5) to $\Omega_{\text {inc }}$, and obtain an integral equation for $u$ of the form

$$
u(\boldsymbol{x})-\int_{\Omega_{\mathrm{inc}}} \Phi(\boldsymbol{x}-\boldsymbol{y}) \nabla \cdot(b(\boldsymbol{y}) \nabla u(\boldsymbol{y})) d A(\boldsymbol{y})=[\Phi * f](\boldsymbol{x}), \quad \boldsymbol{x} \in \Omega_{\mathrm{inc}} .
$$

Our gain is to have converted the equation (3.4), which involved an unbounded operator on an infinite domain, to (3.6), which involves a bounded operator on a finite domain. The integral operator in equation (3.6) is in general not compact, but well-posedness can often be assured via a simple positivity or perturbation argument.

\subsection{A local lattice equation}

To convert the equation (3.1) to an equation on the finite domain $\Omega_{\text {inc }}$, we follow the template established by the continuum case in Section 3.2 and convolve (3.1) by the lattice fundamental solution $\phi$. This yields the equation

$$
[(\mathrm{I}+\mathrm{GB}) u](\boldsymbol{m})=[\mathrm{G} f](\boldsymbol{m}), \quad \boldsymbol{m} \in \Omega_{\text {inc }} .
$$

It is in many environments convenient to post-multiply (3.7) by B again, which results in the equation

$$
(\mathrm{I}+\mathrm{BG}) \mu=\mathrm{BG} f,
$$

where the new unknown variable is $\mu=\mathrm{B} u$. Once (3.8) has been solved for $\mu$, the full solution $u$ is given by the formula

$$
u=\mathrm{G}(f-\mu) .
$$




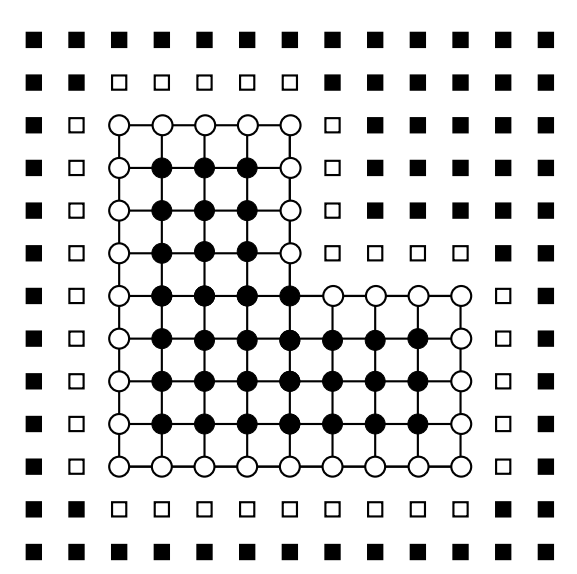

(a)



(b)

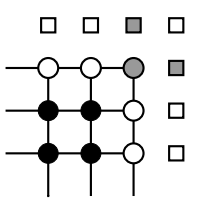

(c)

Figure 4.1: (a) An example of a lattice domain, $\bar{\Omega}=\Gamma \cup \Omega$. The black circles form the interior $\Omega$ and the white circles form the boundary $\Gamma$. (b) Illustration of the set $\mathbb{D}_{m}$ (the grey square) for a boundary node $m$ (grey circle) along a straight edge. (c) Illustration of $\mathbb{D}_{m}$ for a corner node.

\subsection{Numerical methods}

We have found that the equation (3.8) can easily be solved using an iterative solver such as GMRES. For large scale problems, application of the operator $G$ can be accelerated using the lattice FMM described in Section 2.4. The lattice FMM can also be used to rapidly evaluate the potential via (3.9) once $\mu$ has been determined from (3.8).

\section{Techniques for lattice boundary value problems}

In this section we describe techniques for solving the lattice equilibrium equations on a finite lattice with prescribed boundary conditions. The techniques can be modified to a wide range of different boundary conditions (see [11]) but for concreteness, we restrict attention to the basic Dirichlet and Neumann boundary conditions.

\subsection{Problem definition}

\subsubsection{Definition of a lattice domain and boundary}

Let $\bar{\Omega}$ denote a finite subset of $\mathbb{Z}^{2}$. We define the interior of $\bar{\Omega}$ as the set $\Omega$ of nodes whose four neighbors are all contained in $\bar{\Omega}$, and the boundary $\Gamma$ as the remaining nodes, $\Gamma=\bar{\Omega} \backslash \Omega$. Figure 4.1(a) illustrates these definitions. For simplicity, we assume that $\Omega$ forms a connected lattice.

In addition to defining the boundary of the domain, we also need to define exterior and interior boundary flux operators, analogous to normal derivatives in the continuum case. To this end, we let for a given boundary node $\boldsymbol{n} \in \Gamma$ the set $\mathbb{D}_{\boldsymbol{n}}$ denote the set of all points in $\mathbb{Z}^{2}$ that connect to $\boldsymbol{n}$, but are not contained in $\bar{\Omega}$. We let $\mathbb{E}_{\boldsymbol{n}}$ denote the remaining nodes connecting to $\boldsymbol{n}$ so that $\mathbb{D}_{\boldsymbol{n}} \cup \mathbb{E}_{\boldsymbol{n}}$ forms a disjoint union of the four nodes connecting to $\boldsymbol{n}$, see Figure 4.1(b,c). We can now define an exterior difference operator $\partial$ via

$$
[\partial u](\boldsymbol{n})=\sum_{\boldsymbol{k} \in \mathbb{D}_{n}}(u(\boldsymbol{k})-u(\boldsymbol{n}))
$$


and an interior difference operator $\bar{\partial}$ via

$$
[\bar{\partial} u](\boldsymbol{n})=\sum_{\boldsymbol{k} \in \mathbb{E}_{\boldsymbol{n}}}(u(\boldsymbol{n})-u(\boldsymbol{k})) .
$$

\subsubsection{The Dirichlet problem}

A Boundary Value Problem (BVP) with Dirichlet boundary conditions takes the form

$$
\left\{\begin{aligned}
{[\mathrm{A} u](\boldsymbol{m}) } & =0, & & \boldsymbol{m} \in \Omega, \\
u(\boldsymbol{m}) & =g(\boldsymbol{m}), & & \boldsymbol{m} \in \Gamma .
\end{aligned}\right.
$$

It can be demonstrated that (4.3) has a unique solution for any boundary load $g$, see e.g. [8].

\subsubsection{The Neumann problem}

This problem corresponds physically to prescribing the boundary fluxes, rather than the temperatures. Mathematically, we specify the values of the interior difference operator, which results in the equation

$$
\begin{cases}{[\mathrm{A} u](\boldsymbol{m})=0,} & \boldsymbol{m} \in \Omega, \\ {[\bar{\partial} u](\boldsymbol{m})=g(\boldsymbol{m}),} & \boldsymbol{m} \in \Gamma .\end{cases}
$$

When

$$
\sum_{\boldsymbol{m} \in \Gamma} g(\boldsymbol{m})=0
$$

equation (4.4) has a solution that is unique up to a constant.

\subsection{The continuum analog}

The continuum analogs of (4.3) and (4.4) are of course

$$
\left\{\begin{aligned}
{[-\Delta u](\boldsymbol{x}) } & =0, & & \boldsymbol{x} \in \Omega, \\
u(\boldsymbol{x}) & =g(\boldsymbol{x}), & & \boldsymbol{x} \in \Gamma,
\end{aligned}\right.
$$

and

$$
\left\{\begin{aligned}
{[-\Delta u](\boldsymbol{x}) } & =0, & & \boldsymbol{x} \in \Omega, \\
\frac{\partial u(\boldsymbol{x})}{\partial n} & =g(\boldsymbol{x}), & & \boldsymbol{x} \in \Gamma .
\end{aligned}\right.
$$

It is well known from classical potential theory that the solution to (4.6) admits the representation

$$
u(\boldsymbol{x})=\int_{\Gamma} D(\boldsymbol{x}, \boldsymbol{y}) \sigma(\boldsymbol{y}) d \ell(\boldsymbol{y}),
$$

where $D$ is the so called double layer kernel

$$
D(\boldsymbol{x}, \boldsymbol{y})=\frac{\partial}{\partial \boldsymbol{n}(\boldsymbol{y})} \Phi(\boldsymbol{x}-\boldsymbol{y})=\boldsymbol{n}(\boldsymbol{y}) \cdot \nabla_{\boldsymbol{y}} \Phi(\boldsymbol{x}-\boldsymbol{y})=\frac{\boldsymbol{n}(\boldsymbol{y}) \cdot(\boldsymbol{x}-\boldsymbol{y})}{2 \pi|\boldsymbol{x}-\boldsymbol{y}|^{2}},
$$


and $\sigma$ is a boundary potential that can be determined by solving the second kind Fredholm equation

$$
\frac{1}{2} \sigma(\boldsymbol{x})+\int_{\Gamma} D(\boldsymbol{x}, \boldsymbol{y}) \sigma(\boldsymbol{y}) d \ell(\boldsymbol{y})=g(\boldsymbol{x}), \quad \boldsymbol{x} \in \Gamma .
$$

Likewise, any solution to (4.7) admits a representation (up to addition of a constant)

$$
u(\boldsymbol{x})=\int_{\Gamma} S(\boldsymbol{x}, \boldsymbol{y}) \sigma(\boldsymbol{y}) d \ell(\boldsymbol{y}),
$$

where $S$ is the so called single layer kernel

$$
S(\boldsymbol{x}, \boldsymbol{y})=\Phi(\boldsymbol{x}-\boldsymbol{y})
$$

and $\sigma$ is a boundary potential that can be determined by solving the second kind Fredholm equation

$$
-\frac{1}{2} \sigma(\boldsymbol{x})+\int_{\Gamma} D^{*}(\boldsymbol{x}, \boldsymbol{y}) \sigma(\boldsymbol{y}) d \ell(\boldsymbol{y})=g(\boldsymbol{x}), \quad \boldsymbol{x} \in \Gamma
$$

where $D^{*}(\boldsymbol{x}, \boldsymbol{y})$ is the dual kernel of (4.8),

$$
D^{*}(\boldsymbol{x}, \boldsymbol{y})=\frac{\partial}{\partial \boldsymbol{n}(\boldsymbol{x})} \Phi(\boldsymbol{x}-\boldsymbol{y})=\boldsymbol{n}(\boldsymbol{x}) \cdot \nabla_{\boldsymbol{x}} \Phi(\boldsymbol{x}-\boldsymbol{y})=-\frac{\boldsymbol{n}(\boldsymbol{x}) \cdot(\boldsymbol{x}-\boldsymbol{y})}{2 \pi|\boldsymbol{x}-\boldsymbol{y}|^{2}}
$$

We observe that the operator on the left hand side of (4.9) has a one-dimensional null-space, and that its range has co-dimension one (corresponding to the fact that $g$ must integrate to zero). This causes very little difficulty in the construction of numerical methods based on (4.9) since the range is known analytically.

\subsection{Lattice boundary equations}

Inspired by the continuum case, a lattice analog of the double layer potential was proposed in $[8,11]$ (and was briefly mentioned in Section 1 in equation (1.13)). It is obtained by differentiating the fundamental solution $\phi$ using the external difference operator $\partial$ defined in (4.1),

$$
d(\boldsymbol{m}, \boldsymbol{n})=\partial_{\boldsymbol{n}} \phi(\boldsymbol{m}-\boldsymbol{n})=\sum_{\boldsymbol{k} \in \mathbb{D}_{n}}[\phi(\boldsymbol{m}-\boldsymbol{k})-\phi(\boldsymbol{m}-\boldsymbol{n})]
$$

(The subscript $\boldsymbol{n}$ in $\partial_{\boldsymbol{n}}$ simply indicates that the difference operator is acting on the variable $\boldsymbol{n}$.) We define the corresponding operator $\mathrm{D}$ via

$$
[\mathrm{D} q](\boldsymbol{m})=\sum_{n \in \Gamma} d(\boldsymbol{m}, \boldsymbol{n}) q(\boldsymbol{n}) .
$$

It can be shown that any solution $u$ of (4.3) admits the representation

$$
u=\mathrm{D} q
$$


for some boundary charge distribution $q$. An equation for $q$ is obtained by simply restricting (4.13) to $\Gamma$ :

$$
\sum_{n \in \Gamma} d(\boldsymbol{m}, \boldsymbol{n}) q(\boldsymbol{n})=g(\boldsymbol{m}), \quad \boldsymbol{m} \in \Gamma
$$

Both theoretical and numerical results in [11] indicate that the equation (4.14) is typically a well conditioned equation, with the spectrum of $D$ resembling that of a second kind Fredholm operator. (Remark 4.1 presents a calculation that perhaps makes this claim more intuitively plausible.)

Turning next to the Neumann equation (4.4), it can be shown that up to addition of a constant, any solution $u$ admits a representation via a single layer potential

$$
u(\boldsymbol{m})=\sum_{n \in \Gamma} s(\boldsymbol{m}, \boldsymbol{n}) q(\boldsymbol{n}),
$$

where the single layer potential $s$ is defined simply via

$$
s(\boldsymbol{m}, \boldsymbol{n})=\phi(\boldsymbol{m}-\boldsymbol{n}) .
$$

Inserting (4.15) into (4.4), we find that $q$ must satisfy the equation

$$
\sum_{n \in \Gamma} d^{*}(\boldsymbol{m}, \boldsymbol{n}) q(\boldsymbol{n})=g(\boldsymbol{m}), \quad \boldsymbol{m} \in \Gamma,
$$

where $d^{*}$ is the kernel

$$
d^{*}(\boldsymbol{m}, \boldsymbol{n})=\bar{\partial}_{\boldsymbol{m}} s(\boldsymbol{m}, \boldsymbol{n}) .
$$

Again, both theoretical and experimental results indicate that the spectral properties of the system matrix in (4.16) resemble those of its continuum analog (4.9): precisely one singular value is zero, and the remaining ones are clustered relatively closely.

Remark 4.1. The operators in equations (4.14) and (4.16) are in a certain sense complementary. To explicate this relationship, let us note that for a boundary node $\boldsymbol{n}$ we have, for any lattice function $u$,

$$
[\mathrm{A} u](\boldsymbol{n})=\sum_{\boldsymbol{k} \in \mathbb{D}_{\boldsymbol{n}} \cup \mathbb{E}_{\boldsymbol{n}}}(u(\boldsymbol{n})-u(\boldsymbol{k}))=[\bar{\partial} u](\boldsymbol{n})-[\partial u](\boldsymbol{n}) .
$$

Consequently, the kernel of (4.14) satisfies

$$
\left[\partial_{\boldsymbol{n}} \phi\right](\boldsymbol{m}-\boldsymbol{n})=\left[\left(\bar{\partial}_{\boldsymbol{n}}-\mathrm{A}_{\boldsymbol{n}}\right) \phi\right](\boldsymbol{m}-\boldsymbol{n})=\left[\bar{\partial}_{\boldsymbol{n}} \phi\right](\boldsymbol{m}-\boldsymbol{n})-\delta(\boldsymbol{m}-\boldsymbol{n}) .
$$

Inserting (4.17) into (4.14) we obtain the equation

$$
g(\boldsymbol{m})=-q(\boldsymbol{m})+\sum_{\boldsymbol{n} \in \Gamma}\left(\bar{\partial}_{\boldsymbol{n}} \phi(\boldsymbol{m}-\boldsymbol{n})\right) q(\boldsymbol{n}), \quad \boldsymbol{m} \in \Gamma .
$$

Analogously, equation (4.16) is equivalent to the equation

$$
g(\boldsymbol{m})=q(\boldsymbol{m})+\sum_{\boldsymbol{n} \in \Gamma}\left(\partial_{\boldsymbol{m}} \phi(\boldsymbol{m}-\boldsymbol{n})\right) q(\boldsymbol{n}), \quad \boldsymbol{m} \in \Gamma .
$$

A benefit of the formulations (4.18) and (4.19) is that they perhaps make it easier to intuit that (4.14) and (4.16) behave qualitatively like second kind Fredholm equations. 


\subsection{Numerical methods}

The procedure for solving the Dirichlet problem (4.3) that was outlined in Section 4.3 consists of two steps: First (4.14) is solved for the boundary load $q$, and then the actual potential $u$ is evaluated from $q$ via the sum (4.13). The second step is executed numerically by simply applying the fast summation technique of Section 2.4. The linear solve in the first step can be solved using an iterative solver accelerated with the fast summation technique of Section 2.4. In numerical experiments, we found that the iterative solver (in our case GMRES) converged rapidly, as one would expect given that the coefficient matrix in (4.14) is extremely well conditioned [11]. However, one can do even better. It turns out that the system matrix in (4.14) is in fact a Hierarchically Semi-Separable matrix [21, 22, 23, 24], which means that not only can the matrix be applied to vectors in $O\left(N_{\text {boundary }}\right)$ time, but it is possible to directly compute an approximation to its inverse in linear time. We implemented the scheme of [24] and found that a matrix of size 102, $400 \times 102,400$ (corresponding to a lattice with about $6.5 \times 10^{8}$ nodes) could be inverted in 1 minute, and applied to a vector in 1 second. The computational time of course depends on the requested accuracy, and the numbers reported refer to a computation whose relative accuracy was $10^{-10}$. See Section 6 for details.

The Neumann problem (4.4) can be solved numerically using procedures entirely analogous to those described for the Dirichlet problem. The Neumann problem involves the additional complication that the system matrix in (4.16) is singular. However, its range is known analytically (it is the set of functions that sum to zero), so the system can easily be modified to obtain a non-singular well-conditioned equation.

\section{Combined techniques}

\subsection{Problem statement}

In this section, we consider an equation on a bounded domain $\Omega$ for a problem that involves both a body load, and deviations from perfect periodicity. Letting $B$ denote a non-negative operator supported on some subset $\Omega_{\text {inc }}$ of $\Omega$, such an equation takes the form

$$
\left\{\begin{aligned}
{[(\mathrm{A}+\mathrm{B}) u](\boldsymbol{m}) } & =f(\boldsymbol{m}), & & \boldsymbol{m} \in \Omega, \\
u(\boldsymbol{m}) & =g(\boldsymbol{m}), & & \boldsymbol{m} \in \Gamma,
\end{aligned}\right.
$$

where $f$ is a given body load, and $g$ is a given Dirichlet boundary condition. We will convert equation (5.1) to an equation defined on the smaller set $\Gamma \cup \Omega_{\text {inc }}$. The technique is exact and works for any non-negative operator B. However, it is particularly well suited to the case where $\Omega_{\text {inc }}$ is in some sense a "small" subset of $\Omega$, and $f$ is either zero, or also supported on a "small" subset.

\subsection{Reformulation of the difference equation}

We look for a solution of the form

$$
u=\mathrm{G} f-\mathrm{G} \mu+\mathrm{D} q
$$


where $D$ is the double layer operator defined in (4.12), where $q$ is a function on $\Gamma$ that is to be determined, and $\mu$ is the (as yet unknown) function

$$
\mu=\mathrm{B} u \text {. }
$$

It is immediately clear that if (5.2) and (5.3) are satisfied, then for $\boldsymbol{m} \in \Omega$,

$$
[\mathrm{A} u](\boldsymbol{m})=[\mathrm{A} \mathrm{G} f](\boldsymbol{m})-[\mathrm{A} \mathrm{G} \mu](\boldsymbol{m})+[\mathrm{AD} q](\boldsymbol{m})=f(\boldsymbol{m})-\mu(\boldsymbol{m})+0=f(\boldsymbol{m})-[\mathrm{B} u](\boldsymbol{m}),
$$

so the difference equation in (5.1) is satisfied. To enforce the boundary condition, we require $\mu$ and $q$ to satisfy

$$
g(\boldsymbol{m})=[\mathrm{G} f](\boldsymbol{m})-[\mathrm{G} \mu](\boldsymbol{m})+[\mathrm{D} q](\boldsymbol{m}), \quad \boldsymbol{m} \in \Gamma .
$$

It remains to convert (5.2) to an equation that does not involve $u$. This is easily done by applying $\mathrm{B}$ to $(5.2)$ and restricting the resulting equation to $\Omega_{\text {inc }}$,

$$
\mu(\boldsymbol{m})=[\mathrm{BG} f](\boldsymbol{m})-[\mathrm{BG} \mu](\boldsymbol{m})+[\mathrm{BD} q](\boldsymbol{m}), \quad \boldsymbol{m} \in \Omega_{\mathrm{inc}} .
$$

Combining (5.4) and (5.5), we obtain the system

$$
\left[\begin{array}{cc}
\mathrm{D}_{\Gamma} & -\mathrm{G}_{\left(\Gamma \leftarrow \Omega_{\mathrm{inc}}\right)} \\
-\mathrm{B}_{\Omega_{\mathrm{inc}}} \mathrm{D}_{\left(\Omega_{\mathrm{inc}} \leftarrow \Gamma\right)} & \left(\mathrm{I}+\mathrm{B}_{\Omega_{\mathrm{inc}}} \mathrm{G}_{\Omega_{\mathrm{inc}}}\right)
\end{array}\right]\left[\begin{array}{c}
q \\
\mu
\end{array}\right]=\left[\begin{array}{c}
g-\mathrm{G}_{(\Gamma \leftarrow \Omega)} f \\
\mathrm{~B}_{\Omega_{\mathrm{inc}}} \mathrm{G}_{\left(\Omega_{\mathrm{inc}} \leftarrow \Omega\right)} f
\end{array}\right] .
$$

In (5.6) we added subscripts to the operators to indicate their range and domain. For instance, $D_{\Gamma}$ is an operator mapping a function on $\Gamma$ to a function on $\Gamma$ while $\mathrm{D}_{\left(\Omega_{\mathrm{inc}} \leftarrow \Gamma\right)}$ is an operator mapping a function on $\Gamma$ to one defined on $\Omega_{\text {inc }}$.

\subsection{Numerical methods}

All matrices in the linear system (5.6) are amenable to fast schemes for applying a matrix to a vector, and in the examples we have investigated, GMRES converges to a solution reasonably fast. Empirically, we found that the efficiency of the method can be improved if we exploit the fact that an approximation to $D_{\Gamma}^{-1}$ can be computed (see Section 4.4) and directly eliminate the vector $q$ from the system. This results in the equation

$$
\tilde{\mathrm{A}} \mu=h
$$

where

$$
\tilde{\mathrm{A}}=\left[-\mathrm{B}_{\Omega_{\mathrm{inc}}} \mathrm{D}_{\left(\Omega_{\mathrm{inc}} \leftarrow \Gamma\right)} \mathrm{D}_{\Gamma}^{-1} \mathrm{G}_{\left(\Gamma \leftarrow \Omega_{\mathrm{inc}}\right)}+\left(\mathrm{I}+\mathrm{B}_{\Omega_{\mathrm{inc}}} \mathrm{G}_{\Omega_{\mathrm{inc}}}\right)\right]
$$

and

$$
h=\mathrm{B}_{\Omega_{\mathrm{inc}}} \mathrm{G}_{\left(\Omega_{\mathrm{inc}} \leftarrow \Omega\right)} f+\mathrm{B}_{\Omega_{\mathrm{inc}}} \mathrm{D}_{\left(\Omega_{\mathrm{inc}} \leftarrow \Gamma\right)} \mathrm{D}_{\Gamma}^{-1}\left(g-\mathrm{G}_{(\Gamma \leftarrow \Omega)} f\right) .
$$

Once (5.7) has been solved, the vector $q$ is retrieved via the formula

$$
q=\mathrm{D}_{\Gamma}^{-1}\left(g-\mathrm{G}_{(\Gamma \leftarrow \Omega)} f+\mathrm{G}_{\left(\Gamma \leftarrow \Omega_{\mathrm{inc}}\right)} \mu\right)
$$

and then $u$ can be obtained from (5.2). 


\section{Numerical results}

In this section, we illustrate the robustness of the proposed methodology and show that its speed compares favorably to FFT based methods even for moderate problem sizes.

All experiments are run on a Dell desktop computer with 2GB of RAM and an Intel Pentium $43.4 \mathrm{GHz}$ dual processor. The methods were run at a requested relative precision of $10^{-10}$. The techniques were implemented rather crudely in Matlab, which means that significant further gains in speed should be achievable.

\subsection{Numerical results for the lattice Fast Multipole Method}

In this section, we compare two different methods for solving the lattice Poisson equation

$$
[\mathrm{A} u](\boldsymbol{m})=f(\boldsymbol{m}), \quad \boldsymbol{m} \in \Omega,
$$

where $\Omega$ is an $n \times n$ square subdomain of $\mathbb{Z}^{2}$. The first method is the lattice FMM described in Section 2.4, and the second is the FFT. The two methods produce slightly different solutions since they naturally enforce different boundary conditions (the free-space problem (2.1), (2.3) for the FMM, and periodic boundary conditions on $\Omega$ for the FFT), but this is immaterial for our present purposes. We suppose throughout that $n$ is a power of two to make the comparison as favorable to the FFT as possible. We let $T_{\mathrm{ft}}$ denote the time required by the FFT to compute (2.4), and $T_{\text {FMM }}$ the time for the FMM.

In the first experiment, we suppose that every node in the lattice is loaded, so that $N_{\text {source }}=N_{\text {domain }}=n^{2}$. In this case, we expect

$$
T_{\mathrm{fft}} \sim n^{2} \log (n), \quad \text { and } \quad T_{\mathrm{FMM}} \sim n^{2},
$$

and the purpose of the numerical experiment is simply to see how the constants of proportionality compare. Figure 6.1(a) provides the answer. We see that the FMM is slower by roughly one or two orders of magnitude.

In the second experiment, we suppose that $f$ is only sparsely supported in the domain, so that $N_{\text {source }} \ll N_{\text {domain }}$. To be precise, we distributed the loads on a circle inscribed in the square $\Omega$ in such a way that $N_{\text {source }}=\alpha n$ nodes were loaded, for $\alpha=0.25,0.5,0.75$. In this case, we expect

$$
T_{\mathrm{fft}} \sim n^{2} \log (n), \quad \text { and } \quad T_{\mathrm{FMM}} \sim N_{\text {source }} \sim n .
$$

Figure 6.1(b) provides the measured times. It confirms our expectation that $T_{\mathrm{FMM}}$ does not depend on $N_{\text {domain }}$, and indeed, that the FMM can handle a situation with $n=10^{6}$ loaded nodes in a domain involving $N_{\text {domain }}=10^{12}$ lattice nodes.

\subsection{Numerical results for finite lattices}

Next, we investigate the techniques described in Section 4.4, as applied to solving the lattice Dirichlet problem (4.3) on the four domains illustrated in Figure 6.2. The ellipses have an aspect ratio of 0.75 , and the $U$-shapes are scaled so that their thickness is one quarter the length of the long side. 


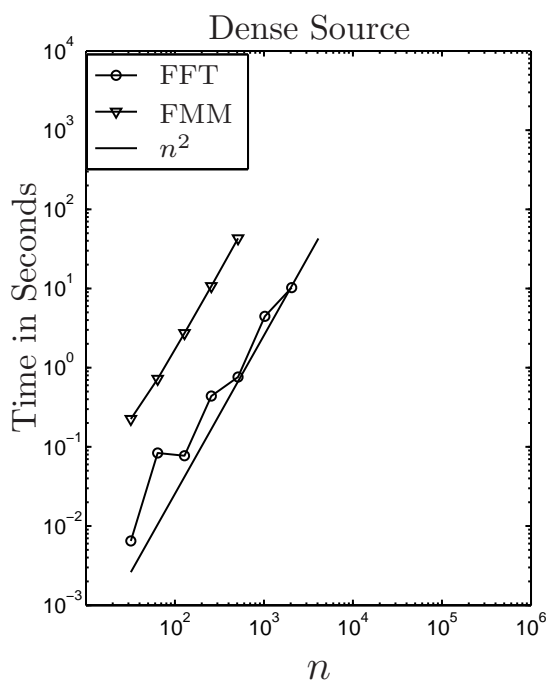

(a)

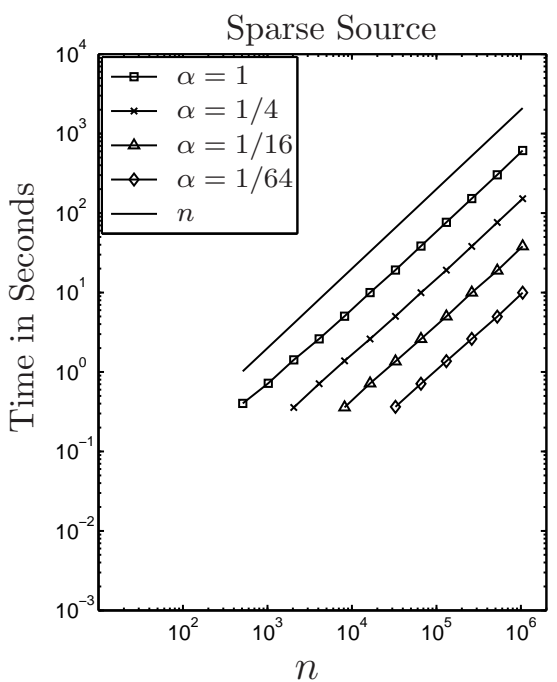

(b)

Figure 6.1: Computational times required for solving (6.1) using the FFT and the FMM.

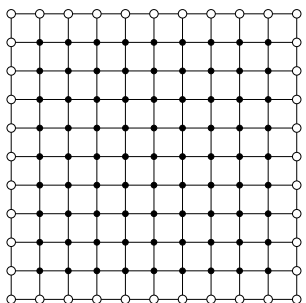

(a) Square

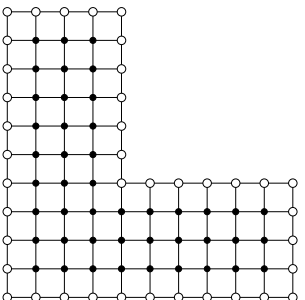

(b) L-shape

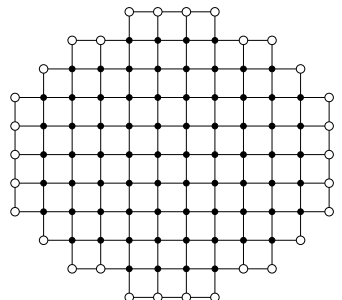

(c) Ellipse

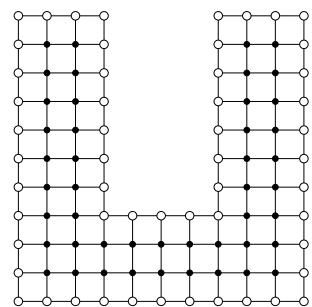

(d) U-shape

Figure 6.2: Finite lattice geometries

For each domain, we recast the BVP (4.3) as a boundary equation of the form (4.14), and then solved (4.14) using the direct solvers described in Section 4.4. A direct solver of this kind involves three steps: (1) Create a compressed representation of the operator in a "data-sparse" format. (2) Compute an approximation to the inverse of the compressed operator. (3) Apply the inverse to the source vector. The times required for each of these three steps are shown in Figure 6.3. We make three observations:

- All steps in the computation scale linearly with the number of points $N_{\text {boundary }}$ on the boundary of the domain.

- The constant of proportionality is small. Specifically, a lattice with $N_{\text {domain }} \approx$ $6.5 \times 10^{8}$ nodes can be processed in one minute. Once the inverse of the boundary operator has been computed, additional solves take only one second.

- Among the three steps of the direct solver, the compression takes by far the longest, and we expect that much could be gained by improving on the crude method we implemented. 

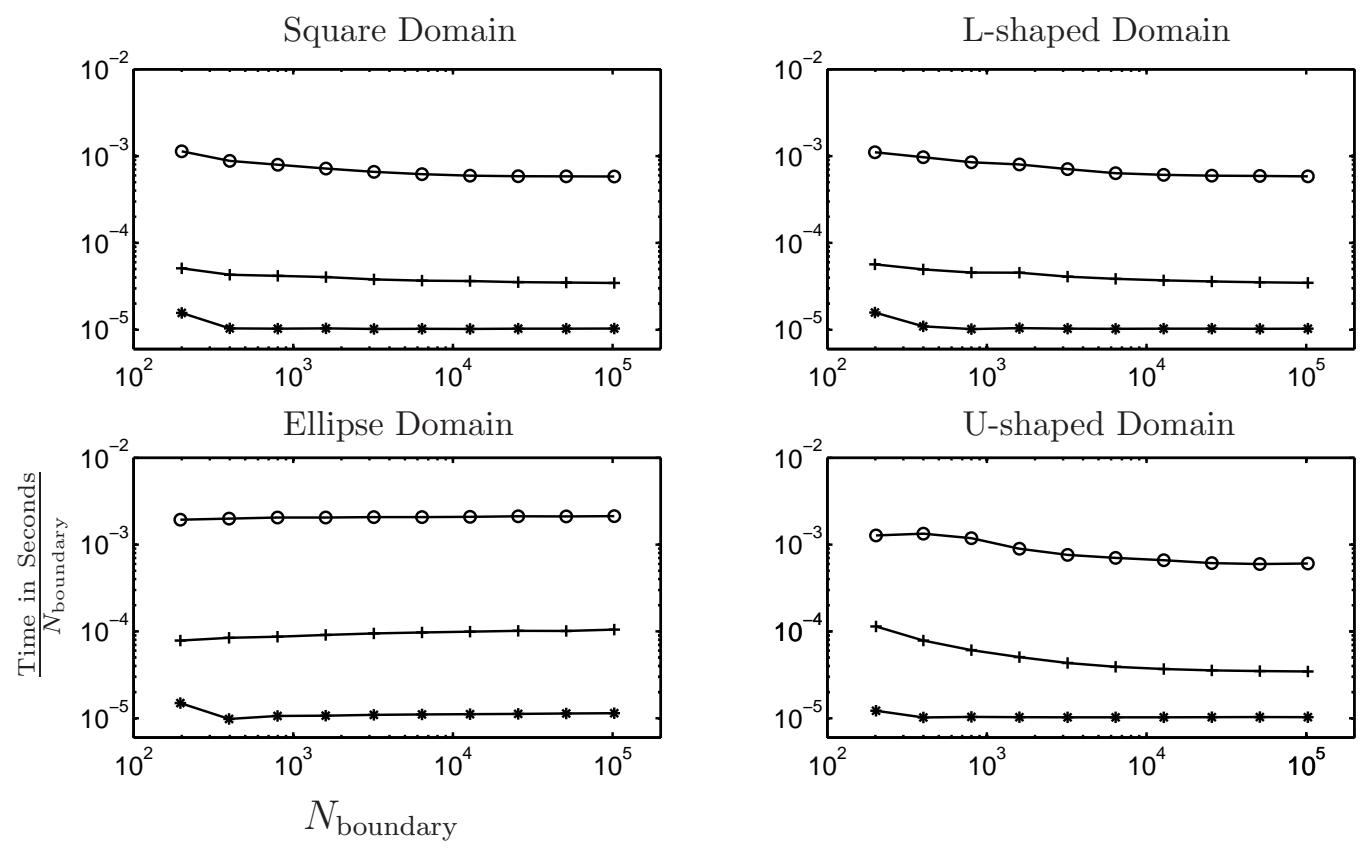

Figure 6.3: Times for inversion of the double layer operator. Labels: -o- Compression, --- Inversion, -*- Application

\subsection{Numerical results for finite lattices with inclusions}

The experiments reported in this section are included to illustrate the conditioning of the linear systems (5.6) and (5.7) that model a finite lattice with local deviations from perfect periodicity. As an examples of such lattices, we consider a sequence of square $79 \times 79$ lattices in which $p$ percent of the internal nodes (chosen at random) had been cut, see Figure 6.4(a). As $p \rightarrow 0$, the condition numbers of (5.6) and (5.7) approach the condition number for the unperturbed boundary equation (4.14), which is excellent (typically less than 10, see [11]). The interesting question is what happens as the lattice gets pushed further away from perfect periodicity.

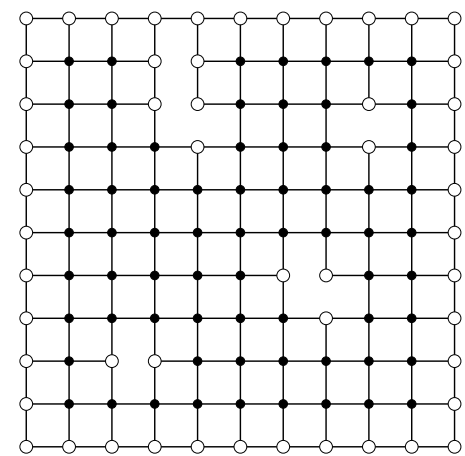

(a)

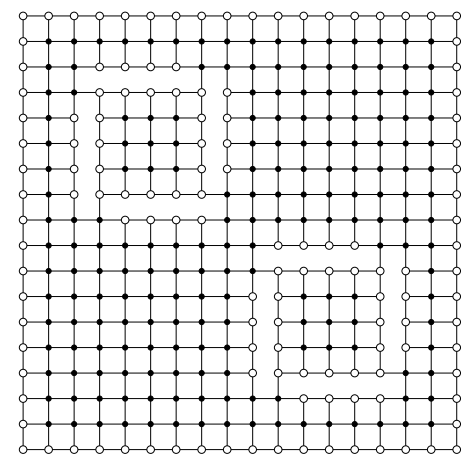

(b)

Figure 6.4: Lattice domains with inclusions

Figure 6.5(a) shows the condition numbers of (5.6) and (5.7) as functions of $p$. 
Figure 6.5(b) shows the closely related graphs illustrating of the number of iterations in GMRES required to attain a residual of less than $10^{-10}$, again as functions of $p$. We first observe that the conditioning remains entirely acceptable for all values of $p$ that we tested. However, between the two formulations, the Schur complement provides significantly better performance.

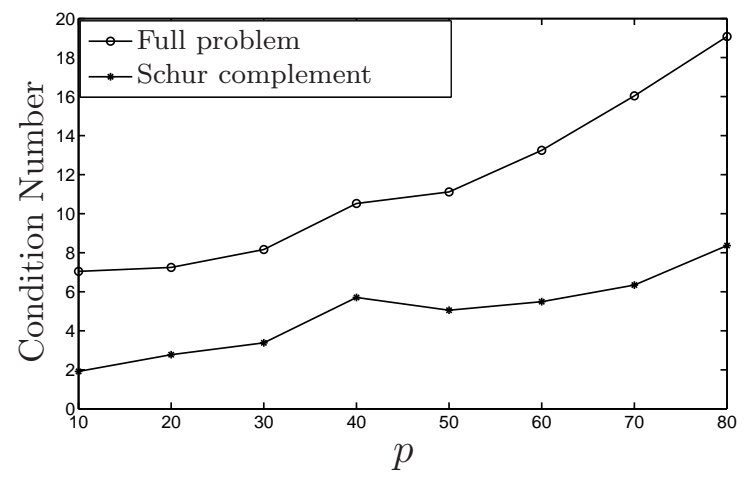

(a)

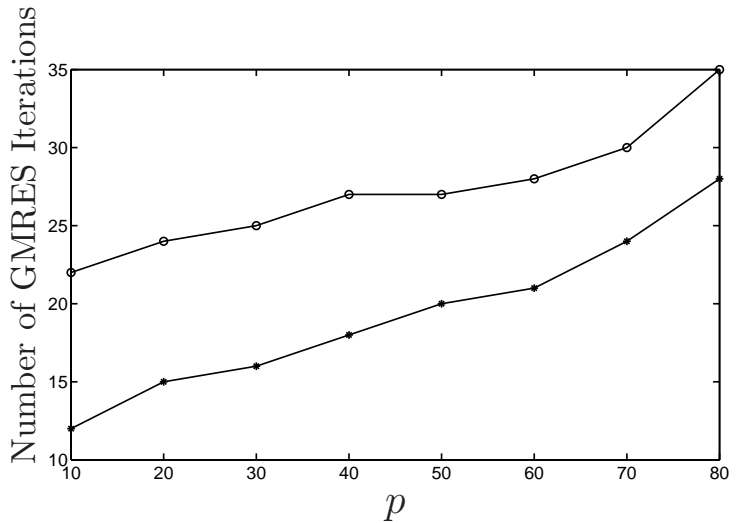

(b)

Figure 6.5: Finite lattice with random inclusions increasing from $20 \%$ to $80 \%$ of the domain. Square size is $79 \times 79$ Labels: -o- Full problem, -+- Schur complement problem

As a final example, we considered a square with $79 \times 79$ nodes, in which two smaller squares were partially separated from the main body of the lattice, as shown in Figure 6.4(b). The separation was accomplished by cutting $p$ percent of the links (chosen at random) on each side of the small squares. We ran experiments all the way up to $p=100$, at which point the interior of each square is connected to the rest of the lattice by only one link at each corner. The potential was grounded at the boundary (i.e. a homogeneous Dirichlet boundary condition was enforced), and two point sources were placed at the center of each small square. The solution technique described in Section 5.3 handled every value of $p$ with ease. The potential fields associated with some values of $p$ are shown in Figure 6.6. We remark that for large values of $p$, the physics of the underlying problems is quite ill-conditioned, and that the problems considered would be hard for previously existing methods.

\section{Generalization to other lattice operators}

While this manuscript has focused exclusively on lattice equations involving the discrete Laplace operator (1.1) acting on the square lattice $\mathbb{Z}^{2}$ (or subsets thereof), the methods can straightforwardly be generalized in several ways.

The extension to other lattice operators on $\mathbb{Z}^{2}$ is particularly simple. The methods for the free-space problem, and for lattices with inclusions, apply directly once a fundamental solution for the operator under consideration has been constructed. Techniques for constructing such fundamental solutions are described in [12]. The techniques for handling boundary conditions in Section 4 also generalize, with the only caveat that for difference operators that involve more than the eight nearest 
neighbors of any lattice node, the boundary of a lattice domain must be extended to a boundary layer of nodes, sufficiently wide that the nodes inside the layer do not communicate directly with the nodes on the outside.

The extension to operators on more general mono-atomic or multi-atomic periodic lattices in two dimensions (such as triangular and hexagonal) is also relatively straightforward [8].

The extension to lattices in three dimensions is in principle not hard either, although in this case iterative methods must be used to solve the boundary equations that generalize (4.14), since we do not currently have methods for computing the inverse of such a boundary operator in linear time. Moreover, the FMM that we used would probably not perform well in three dimensions, and we would recommend the use of the method of [20].

\section{Conclusions}

The paper describes efficient techniques for solving elliptic difference equations on lattice domains. For simplicity of presentation, the paper focuses on lattice equations involving the discrete Laplace operator (1.1) acting on the square lattice $\mathbb{Z}^{2}$, or subsets thereof. Discrete analogs to boundary integral equations are proposed. These equations are amenable to fast solvers such as the Fast Multipole Method. Techniques are introduced for problems involving inclusions or local deviations from perfect periodicity. The complexity of the proposed method is $O\left(N_{\text {boundary }}+N_{\text {source }}+N_{\text {inc }}\right)$ where $N_{\text {boundary }}$ is the number of nodes on the boundary of the domain, $N_{\text {source }}$ is the number of nodes subjected to body loads, and $N_{\text {inc }}$ is the number of nodes that deviate from perfect periodicity.

Numerical experiments that demonstrate the robustness, versatility, and speed of the methods were presented. For instance, it was demonstrated that using a standard desktop PC, a lattice Poisson equation on a lattice with $N_{\text {domain }}=10^{12}$ nodes, of which $N_{\text {source }}=10^{6}$ were loaded, was solved to ten digits of accuracy in three minutes. It should be noted that this problem is about six orders of magnitude larger than the largest Poisson problem that can be handled via the FFT. However, our experiments also indicate that for problems in which a majority of the nodes were loaded (so that the quotient $N_{\text {source }} / N_{\text {domain }}$ is not small), FFT based methods are much faster. The experiments also demonstrate that using lattice equivalents of boundary integral equations along with fast methods for dense matrices, it is possible to solve a boundary value problem on a lattice with $6.5 \cdot 10^{9}$ nodes (for which $N_{\text {boundary }}=25600$ ) in 1 minute for the first solve; again using a standard desktop PC. The solution was accurate to ten digits. Once the first problem has been solved, additional right hand sides (that is, problems specifying other values on the boundary) can be handled in 1 second.

Acknowledgements: Parts of the work described was inspired by suggestions from Professors Ivo Babuška and Gregory J. Rodin at the University of Texas at Austin. The work was supported by the NSF (DMS grants \#0748488 and \#0610097), the Army Research Office (DAAD 19-99-1-014), and by the Wallenberg Foundation. 


\section{References}

[1] M. Ernst, P. Van Velthoven, Random walks on cubic lattices with bond disorder, Journal Journal of Statistical Physics 45 (1986) 1001-1030.

[2] T. Degrands, C. De Tar, Lattice Methods for Quantum Chromodynamics, World Scientific Publishing Company, Hackensack, NJ, 2006.

[3] I. Montvay, G. Mnster, Quantum Fields on a Lattice, Cambridge Monographs on Mathematical Physics, Cambridge University Press, Cambridge, 1997.

[4] E. Schlangen, E. J. Garboczi, New method for simulating fracture using an elastically uniform random geometry lattice, Int. J. Engng. Sci. 34 (1996) 11311144.

[5] H. A. Herrmann, H. J., S. Roux, Fracture of disordered, elastic lattices in two dimensions, Phys. Rev. B 39 (1989) 637-648.

[6] V. Deshpande, N. Fleck, M. Ashby, Effective properties of the octet-truss lattice material, Journal of the Mechanics and Physics of Solids 49 (2001) 1747 - 1769.

[7] Y. Wang, P. Mora, Macroscopic elastic properties of regular lattices, J. Mech. Phys. Solids 56 (2008) 2459-3474.

[8] P. Martinsson, Fast multiscale methods for lattice equations, Ph.D. thesis, University of Texas at Austin, Computational and Applied Mathematics, 2002.

[9] J. C. Wallach, L. J. Gibson, Mechanical behavior of a three-dimensional truss material, International Journal of Solids and Structures 38 (2001) 7181 - 7196.

[10] K. Atkinson, The numerical solution of integral equations of the second kind, Cambridge University Press, Cambridge, 1997.

[11] P. Martinsson, G. J. Rodin, Boundary algebraic equations for lattice problems, Proc. R. Soc. Lond. Ser. A Math. Phys. Eng. Sci. 465 (2009) 2489-2503.

[12] P. Martinsson, G. Rodin, Asymptotic expansions of lattice green's functions, Proc. Royal Soc. A 458 (2002) 2609 - 2622.

[13] R. J. Duffin, Discrete potential theory, Duke Math. J. 20 (1953) 233-251.

[14] R. J. Duffin, E. P. Shelly, Difference equations of polyharmonic type, Duke Math. J. 25 (1958) 209-238.

[15] A. A. Maradudin, E. W. Montroll, G. H. Weiss, R. Herman, H. W. Milnes, Green's functions for monatomic simple cubic lattices, Acad. Roy. Belg. Cl. Sci. Mém. Coll. in-4 deg. (2) 14 (1960) 176.

[16] L. Greengard, V. Rokhlin, A fast algorithm for particle simulations, J. Comput. Phys. 73 (1987) 325-348. 
[17] L. Greengard, V. Rokhlin, A new version of the fast multipole method for the Laplace equation in three dimensions, in: Acta numerica, 1997, volume 6 of Acta Numer., Cambridge Univ. Press, Cambridge, 1997, pp. 229-269.

[18] Z. Gimbutas, V. Rokhlin, A generalized fast multipole method for nonoscillatory kernels, SIAM J. Sci. Comput. 24 (2002) 796-817 (electronic).

[19] P. Martinsson, V. Rokhlin, An accelerated kernel independent fast multipole method in one dimension, SIAM Journal of Scientific Computing 29 (2007) 1160-11178.

[20] L. Ying, G. Biros, D. Zorin, A kernel-independent adaptive fast multipole algorithm in two and three dimensions, J. Comput. Phys. 196 (2004) 591-626.

[21] S. Chandrasekaran, M. Gu, X. Li, J. Xia, Superfast multifrontal method for large structured linear systems of equations, SIAM J. Matrix Anal. Appl. 31 (2009) 1382-1411.

[22] S. Chandrasekaran, M. Gu, X. Li, J. Xia, Fast algorithms for hierarchically semiseparable matrices, Numer. Linear Algebra Appl. (2010).

[23] S. Chandrasekaran, M. Gu, W. Lyons, A fast adaptive solver for hierarchically semiseparable representations, Calcolo 42 (2005) 171-185.

[24] P. Martinsson, V. Rokhlin, A fast direct solver for boundary integral equations in two dimensions, J. Comp. Phys. 205 (2005) 1-23. 



Figure 6.6: Potential for finite lattice with 2 random square inclusions and homogeneous boundary conditions. Lattice domain size is $79 \times 79$. 